\title{
MAC layer Cooperation Analysis in Cognitive Wireless Networks
}

\author{
Ferran Adelantado \\ Universitat Oberta de \\ Catalunya \\ ferranadelantado@uoc.edu
}

\author{
Angel A. Juan \\ Universitat Oberta de \\ Catalunya \\ ajuanp@uoc.edu
}

\author{
Christos Verikoukis \\ Centre Tecnològic de \\ Telecomunicacions de \\ Catalunya \\ cveri@cttc.es
}

\begin{abstract}
Cognition and cooperation are two concepts closely connected in Cognitive Wireless Networks. In such distributed networks, where there is not any central node responsible for retrieving/forwarding the information from/to all the users, the monitoring of the spectrum availability must be carried out also in a distributed fashion. However, there exists a trade-off between the amount of monitoring information shared among the users and the overhead introduced to the network, particularly when users are equipped with a single antenna. As primary users may suddenly transmit on any licensed channel, all secondary users should monitor periodically alternative idle channels where to resume the ongoing communications in case such a situation occurs. In this paper we analyze the minimum spectrum monitoring frequency required for users to have a number of alternative idle channels.
\end{abstract}

\section{INTRODUCTION}

Traditionally, the radio spectrum has been divided into bands, each one assigned to a specific system and with the appropriate bandwidth to accomplish its requirements. However, some bands of the spectrum have been left unlicensed and only regulated in terms of maximum transmission power and interference constraints [1]. Such fixed-spectrum assignment schemes have been shown to be inefficient [2] but its simple implementation and the relative availability of new unused bands did not justify any reconsideration of such a policy.

The huge demand of new wireless services with high bandwidth requirements along with the difficulty to further increase the carrier frequency have set out a new frequency reutilization challenge. In this context, the term Cognitive Network denotes the networks that use the spectrum bands assigned to other systems when they are detected to be idle. Nowadays, these networks have arisen as a promising solution to cope with the aforementioned spectrum scarcity.

We can distinguish two types of users: the primary or li- censed users (PU) and the secondary or unlicensed users (SU). The spectrum band is completely assigned to PUs; however, secondary users may reuse the spectrum as far as it is idle and provided that they do not incur interference on the primary transmissions. Thereby, SUs are allowed to use the spectrum opportunistically, though they must vacate it as soon as PUs activity is detected. In case a PU transmission is detected, SUs must stop transmitting and resume the ongoing transmission on a different idle channel.

In Cognitive Wireless Networks the spectrum availability detection must rely on the veracity and accuracy of the collected spectrum information. Therefore, insufficient information or false alarms (detection of PU activity when the band is actually idle) cause underutilization of resources. On the other hand, if a network fails to detect PUs, the main condition of cognitive networks is not accomplished and the primary network is interfered by secondary transmissions. In order to overcome this problem two approaches have been proposed [3]. In the first one the primary network is charged to broadcast to SUs the required information. The second approach is based on spectrum sensing through either direct or cooperative distributed sensing, where SUs not only sense the spectrum channels but also share the information with neighbors.

Focusing on distributed secondary networks, the number of transceivers with which each user is equipped plays a crucial role in the performance of the network. When users are equipped with multiple transceivers, one of them is usually devoted to the common control channel and has also the responsibility to exchange the related spectrum sensing information. In the case of a single transceiver, the available time of its operation should be shared between the transmission of data, the transmission of control information and the sensing process. In fact, there exists a trade-off between the amount of monitoring and sensing information shared among the users and the overhead introduced to the network.

Since most of the commercial wireless equipments have a single transceiver per spectrum range, a realistic cognitive analysis should take into account this constraint.

In this study all SUs are assumed to sense the spectrum with the same periodicity and, therefore, to schedule a single sensing process during a sensing period, which will be defined in Section 3. After sensing the spectrum, the information is 
shared with the rest of the users. The mechanism used to share the information and to coordinate the transmissions is out of the scope of this study.

As the cooperative sensing process consumes part of the scarce resources leased from the primary network, the secondary network will increase the spectrum efficiency as long as such resource consumption is reduced, i.e. less frequent cooperative sensing processes. However, a minimum sensing periodicity is required so as to monitor alternative idle channels to resume the ongoing communications in case a PU is detected.

In this context the paper provides an analytical model to compute the minimum sensing periodicity according to the number of sensed channels and SUs in the network. The MAC layer protocol implemented in the SUs is responsible for guaranteeing the required number of available channels. This number of channels is a MAC protocol design parameter and may determine its robustness.

In the current context, where the demand for wireless broadband access has experienced a spectacular growth, guaranteeing a minimum radio resources availability level is necessary to accomplish the Quality of Service (QoS) requirements of several multimedia applications. In such networks where the capacity of the channels is limited and the possibility of being forced to vacate them and resume transmissions on alternative channels is high, multi-channel MAC protocols are a must. Therefore, the study of the sensing process that provides such protocols with the required information is a previous and necessary step. For instance, if not enough resources are found, the constraints on the multimedia services that could be implemented would become more stringent.

The rest of the paper is organized as follows. In Section 2 some related work is stated. Section 3 describes in detail the network model, while Section 4 analyzes the cooperative sensing process. Section 5 shows an example and discusses the results. Finally Section 6 concludes the paper.

\section{RELATED WORK}

Due to the challenges posed by the sensing and usage of spectrum licensed to primary networks, several works have been presented in the literature. Two interesting studies faced this problem from different perspectives $[4,5]$. In the former, the sensing process was modeled as a scheduling problem by making use of previous scheduling works and including the virtual sensing node concept. On the other hand, the latter stated the optimal stopping problem. In the sensing process there exists a trade-off between the number of required sensing periods and the drop of the traffic transmission rate. The more often sensing periods are scheduled, the better the knowledge of the scenario is, but the lower the mean transmission rate becomes. According to the trade-off, Jia et al. described a method where transmitter and receiver agreed on the number of sensing periods to maximize the objectives [5].

The basis of cognitive networks is the utilization, when available, of licensed spectrum bands. This characteristic implies that setting up a common control channel may not always be possible. The varying environment along with the activity of PUs does not allow to assure the existence of a common channel. For instance, Jun et al. showed in [6] the scarce feasibility of assuming a common control channel, probing that this kind of common channel could only be achieved in small areas. Following these conclusions, in [7] a decentralized opportunistic spectrum allocation ad hoc network for secondary users was developed. There was no common channel and it was based on clusters formation in order to take advantage of the spectrum similarity.

\section{NETWORK MODELING}

The secondary cognitive network is composed of a set of SUs that cooperate each other. For a generic SU $u_{i}$, its environment is characterized by the set of primary channels $(M)$ that must be sensed to determine whether they can be used or not.

$$
M=\left\{m_{j}: j>0\right\}
$$

Each channel $m_{j}$ is available with a probability $P_{i d l e}$. The channel is modeled as an ON-OFF process that can be in idle $(\mathrm{OFF})$ or busy $(\mathrm{ON})$ state. Analogously to the so-called coherence time, $T_{U}$ is defined as the average time for which a spectrum channel remains in the idle state. The set of neighboring users $\left(V_{i}\right)$ is composed of all the users within the coverage area of $u_{i}$.

$$
V_{i}=\left\{u_{k}: k \neq i, l\left(u_{k}\right) \in \Omega_{i}\right\}
$$

where $l\left(u_{k}\right)$ stands for the location of user $u_{k}$ and $\Omega_{i}$ for the coverage area of $u_{i}$. The number of neighbors is then the cardinality of the set, written $\left|V_{i}\right|$. All the users are assumed to have the same sensing periodicity. In particular, each user senses the spectrum and transmits the collected information once every $T_{P}$ seconds, named sensing period.

Our analysis is focused on the sensing of a SU, which includes the discovery and the update of the spectrum availability. Only the mentioned processes are modeled, since these processes consume part of the available resources and diminish the effective data capacity.

In order to highlight the effect of the parameters under study, transmissions are assumed to be error-free and the received power levels suffice to decode the packets. Likewise, SUs detect PUs transmissions whenever they occur.

The sensing process of user $u_{i}$ consists in sensing the spectrum and transmitting the obtained information to the neighboring users. Thereby, for $u_{i}$ the spectrum availability information can be obtained by sensing the spectrum and by receiving the information collected by the neighboring users.

There are two important aspects for each user, both of them related to the reliability of the information. On one hand, the user has to rely on the data received from neighbors in order to get benefit from cooperation. On the other hand, the related information should be refreshed periodically in order to get the most possible accuracy. In this proposal the user $u_{i}$ is modeled as a vector $Q_{i}=\left(q_{i, 1}, q_{i, 2}, \ldots, q_{i,|M|}\right)$ that has binary elements $q_{i, j} \in B=\{0,1\}$. Each element $q_{i, j}$ of the vector is associated with a primary system channel $m_{j}$. 
Accordingly, when $u_{i}$ detects the availability of $m_{j}$, by either sensing $m_{j}$ or receiving neighbor's information, $q_{i, j}=1$. If no information on $m_{j}$ is available, or $m_{j}$ is detected busy, or the available information is out-of-date, $q_{i, j}=0$. Information is deemed out-of-date at instant $t$ if it was acquired before $t-T_{U}$.

\section{THE COOPERATIVE SENSING PROCESS ANALYSIS}

When the sensing process is triggered at user $u_{i}$, a set of frequency channels are selected and sensed sequentially. The number of channels sensed in each process is $\phi_{T}$. Each of these channels is sensed during a time $T_{C h \text { Sensed }}$ and the time required for a transciever to switch from channel to channel is $T_{\text {switch }}$. Accordingly, the time spent to sense the range of $\phi_{T}$ channels, $T_{\text {Totalsense }}$, is

$$
T_{\text {TotalSense }}=\left(T_{\text {ChSensed }}+T_{\text {switch }}\right) \cdot \phi_{T}
$$

The number of channels detected to be idle is a subset of the $\phi_{T}$ sensed channels. In this case, the information on the availability of $\phi_{T}$ channels is available at user $u_{i}$ as a consequence of the sensing. Next, the information becomes available at the rest of the $\left|V_{i}\right|$ neighbors because $u_{i}$ broadcasts it. The channel used by user $u_{i}$ to broadcast the information is out of the scope of the paper and depends on the MAC layer protocol design. However, it is worth mentioning that information may be transmitted over a channel licensed to SUs [8], over the ISM band [9] or even over an idle primary channel, though this is still an open issue.

At the MAC layer, the packets devoted to share the information are of fixed length, with header and payload. The time required to transmit these packets, $T_{\text {packet }}$, is then

$$
T_{\text {packet }}=T_{\text {header }}+T_{\text {channel }} \cdot \phi_{T}+T_{\text {other }}
$$

where $T_{\text {header }}$ is the time needed to transmit the header, $T_{\text {channel }}$ is the time required to transmit the information on a single channel and $T_{\text {other }}$ is the time required to transmit other information piggybacked in the MAC packet. It is worth noting that each packet is assumed to have the same header regardless of $\phi_{T}$, and this is the reason why only $T_{\text {channel }}$ is affected by the term $\phi_{T}$. Thereby, the MAC packet devoted for such a purpose has a fixed length part and a part with a length that depends on the number of sensed channels, $\phi_{T}$ (see Figure 1).

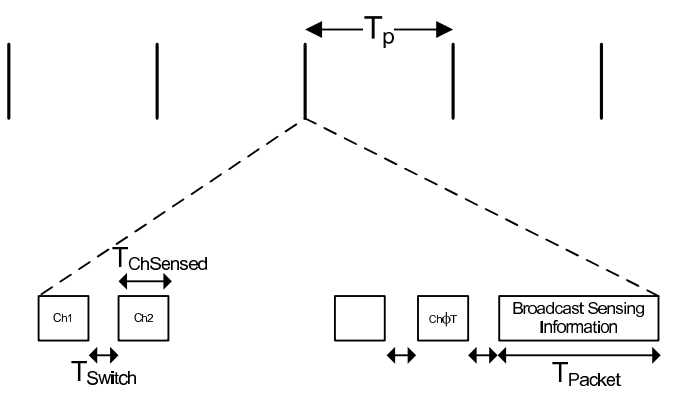

Figure 1: User $u_{i}$ sensing process.

\subsection{Channel availability}

In this section we focus our analysis on a generic channel $m_{j}$. As mentioned in the previous sections, the vector $Q_{i}$ at user $u_{i}$ contains an element which describes the knowledge on the availability of $m_{j}$, i.e. $q_{i, j}$. In particular, $q_{i, j}=1$ implies that the channel was found idle the last time it was sensed and, moreover, this information is deemed up-to-date.

According to the previous sections, the information on the availability of $m_{j}$ retrieved by $u_{i}$ may be seen as an arrival. Likewise, the information transmitted by the neighboring users may also be seen as an arrival. The sum of arrivals is then an arrival process. This arrival process is generated in a distributed fashion by all the users. In fact, the process is in turn the sum of $\left|V_{i}\right|+1$ processes, each one generated by a single source, i.e. $u_{i}$ and the $\left|V_{i}\right|$ neighbors. The sensing processes at each user are scheduled in a TDMA fashion and owing to cooperation there is not any contention among the cooperative users.

In order to gain insight into the availability of channel $m_{j}$ at user $u_{i}$, i.e. $q_{i, j}$, it is essential to characterize the arrival process. It is worth noting that every time an arrival occurs at user $u_{i}$, it only contains information on $\phi_{T}$ channels. Thus, only $\phi_{T}$ elements of $Q_{i}$ are updated. The updating of $q_{i, j}$ is then considered a $q_{i, j}$ arrival. Let $T$ be the time until next arrival occurs at element $q_{i, j}$. The cumulative distribution function $F_{T}(t)$ is expressed as

$$
F_{T}(t)=P(T \leq t)
$$

As no specific order for arrivals is assumed a priori, the arrival for $q_{i, j}$ may be generated by any of the users. If all the users are indexed from 1 to $\left|V_{i}\right|+1, F_{T}(t)$ can be written as

$$
F_{T}(t)=P\left(\bigcup_{x=1}^{\left|V_{i}\right|+1} A_{x}\right)
$$

where $A_{x}=\left\{T_{x} \leq t\right\} . \quad T_{x}\left(1 \leq x \leq\left|V_{i}\right|+1\right)$ is the time until the first $q_{i, j}$ arrival generated by the user $u_{x}$ occurs. According to the inclusion-exclusion principle,

$$
F_{T}(t)=\sum_{k=1}^{\left|V_{i}\right|+1}(-1)^{k-1} \cdot\left(\begin{array}{c}
\left|V_{i}\right|+1 \\
k
\end{array}\right) \cdot P\left(A_{X}\right)
$$

where $A_{X}:=\bigcap_{x \in X} A_{x}$ for every $X \subset\left\{1, \ldots,\left|V_{i}\right|+1\right\}$ with $|X|=k$. Alternatively, by using the Bayes theorem, $P\left(A_{X}\right)$ may be rewritten as

$$
P\left(A_{X}\right)=\prod_{x=1}^{k} P\left(A_{x} / \bigcap_{n=1}^{k-1} A_{n}\right)
$$

This is the general expression for the problem. Yet, in case each user schedules the sensing process independently, the variables $T_{x}$ are independent and $F_{T}(t)$ may be arranged and calculated as

$$
F_{T}(t)=1-\prod_{x=0}^{\left|V_{i}\right|}\left(1-F_{T_{x}}(t)\right)
$$


where $F_{T_{x}}(t)$ stands for the cumulative distribution function of $T_{x}$. As not all the sensing processes triggered by user $u_{x}$ result in a $q_{i, j}$ arrival, let define $\tilde{T}_{x}\left(0 \leq \tilde{T}_{x} \leq T_{P}\right)$ as the time until the next sensing process is triggered by user $u_{x}$, no matter if $m_{j}$ is sensed or not. Then,

$$
F_{T_{x}}(t)=\sum_{w=1}^{\infty} F_{\tilde{T_{x}}}\left(t-(w-1) \cdot T_{P}\right) \cdot P(W=w)
$$

where $W$ is a random variable that indicates the number of sensing processes triggered by user $u_{x}$ required to generate a $q_{i, j}$ arrival, i.e. to sense the channel $m_{j}$. For instance, and in order to illustrate the meaning of $W$, if during the second sensing process at $u_{x}$ the channel $m_{j}$ is sensed, $W=2$.

Finally, by definition, the probability density function $f_{T}(t)$ is obtained by deriving $F_{T}(t)$.

As described in Section 3, when user $u_{i}$ receives information on channel $m_{j}, q_{i, j}$ is set to 1 in case the channel is detected idle and $q_{i, j}$ is set to 0 in case it is detected busy. Additionally, if the time elapsed between two consecutive $q_{i, j}$ arrivals exceeds $T_{U}, q_{i, j}$ is also set to 0 .

If $\tau_{1}$ is defined as the time during which $q_{i, j}$ remains 1 between two arrivals at $q_{i, j}$, the probability $P\left(q_{i, j}=1\right)$ is computed as

$$
P\left(q_{i, j}=1\right)=\frac{E\left[\tau_{1}\right]}{E[T]}
$$

where $E[\cdot]$ operator is the expected value. The probability that a channel is found idle is denoted by $P_{\text {idle }}$. As for $\tau_{1}$ calculation, $q_{i, j}$ will be 1 if $m_{j}$ was idle the last time it was sensed and the time between two consecutive sensings of $m_{j}$ is shorter than $T_{U}$. Then,

$$
\begin{gathered}
E\left[\tau_{1}\right]=P_{\text {idle }} \int_{0}^{T_{U}} t f_{T}(t) d t+P_{\text {idle }} \int_{T_{U}}^{\infty} T_{U} f_{T}(t) d t \\
E[T]=\int_{0}^{\infty} t f_{T}(t) d t
\end{gathered}
$$

Arranging (11) the probability $P\left(q_{i, j}=1\right)$ results in

$$
P\left(q_{i, j}=1\right)=P_{\text {idle }} \cdot\left(1+\frac{\int_{T_{U}}^{\infty}\left(T_{U}-t\right) f_{T}(t) d t}{\int_{0}^{\infty} t f_{T}(t) d t}\right)
$$

\subsection{Number of available channels}

For a given time instant, the number of available channels for user $u_{i}$ will be the squared norm of the vector $Q_{i}$

$$
I_{i}=\left\|Q_{i}\right\|^{2}=\sum_{j=1}^{|M|} q_{i, j}^{2}
$$

The average number of elements equal to 1 for user $u_{i}$ is given by

$$
\bar{I}_{i}=\sum_{z=0}^{|M|} z \cdot C_{z}^{|M|} \cdot P\left(q_{i, j}=1\right)^{z}\left(1-P\left(q_{i, j}=1\right)\right)^{|M|-z}
$$

where $C_{z}^{|M|}$ is the binomial coefficient, which is defined as the number of combinations of $z$ elements out of a set of $|M|$ elements. In the case under study, and assuming that $z$ elements of $Q_{i}$ are set to $1, C_{z}^{|M|}$ is the number of possible combinations of the $z$ available channels. It is then calculated as

$$
C_{z}^{|M|}=\left(\begin{array}{c}
|M| \\
z
\end{array}\right)=\frac{|M| !}{z !(|M|-z) !}
$$

In cognitive networks, the SUs should be able to maintain a minimum $\bar{I}_{i}$ level to guarantee the possibility of switching to alternative channels in case the current channel is suddenly occupied by a PU. When an ongoing communication falls due to the detection of a PU transmission, the SU must resume the secondary communication on another frequency channel. The selection of the new channel implies an inherent delay, though it may be reduced if information on alternative channels has been updated periodically. Thus, the more available alternative channels are monitored, the higher the probability of delay reduction will be. Furthermore, another reason for maintaining a minimum $\bar{I}_{i}$ level is, for instance, the possibility of parallel transmissions when not all users are involved in the same communication.

According to the obtained expressions, for a given number of neighbors $\left|V_{i}\right|, \bar{I}_{i}$ may be increased by raising the period $T_{P}$. However, there exists a trade-off between the sensing process frequency and the time available for data. In other words, the more accurate the knowledge of the environment is, the more limited the data capacity becomes.

\subsection{Limitations of the model}

It is worth noting that the expression derived in (16) do not consider the time devoted to sense the spectrum and share the information. Specifically, the model assumes that the sensing process is a discrete time arrival process, and therefore it is not time consuming.

As shown in (3) and (4), the sensing process of a single user lasts $T_{\text {TotalSensed }}+T_{\text {packet }}$. Let define $T_{\text {Sensing }}$ as the total amount of time devoted by all the users to the sensing within a period $T_{P}$.

$$
T_{\text {Sensing }}=\left(T_{\text {TotalSensed }}+T_{\text {packet }}\right) \cdot\left(\left|V_{i}\right|+1\right)
$$

The model presented will only be valid if $T_{\text {Sensing }}<<T_{P}$. For comparison purposes the normalized time devoted to data and MAC protocol packets is defined as the ratio $r_{A}$. Therefore, the validity of the model may be assumed if $r_{A}$ is close to 1 .

$$
r_{A}=1-\frac{T_{\text {Sensing }}}{T_{P}}
$$

The modeled sensing process is aimed at discovering and monitoring the available resources, and therefore any MAC 
protocol should minimize the consumption of resources for that purpose so as not to diminish the effective data capacity. Accordingly, $r_{A} \sim 1$ can be assumed.

\section{DISCUSSION}

The convenience of monitoring periodically the spectrum to detect alternative idle channels has been stated in the previous sections. Moreover, the expression for the average number of channels has been derived in (16).

According to the analysis carried out in Section 4, the average number of idle channels is highly dependent on the distribution of the sensing process of each user.

The MAC protocol used to share the sensing information is out of the scope of the study. Yet, as several of the parameters involved in the computations are MAC protocol specific, a particular example is proposed.

In order to validate the model and gain insight into the minimum sensing periodicity required to have an average number of idle channels at user $u_{i}$ disposal, $T_{P}$ and $\bar{I}_{i}$ respectively, both theoretical and Monte Carlo results have been obtained.

The proposed example considers that, once the secondary network has been set up, all the users negotiate the sensing periodicity, $T_{P}$, and coordinate the sensing processes in a way such that two or more users are not allowed to sense the spectrum simultaneously. Therefore, all the SUs are allowed to schedule the sensing process with the sole constraint of coordination to avoid simultaneity. In this particular example, we consider that all users schedule the sensing process with the same probability within the sensing period, i.e. $\tilde{T}_{x}$ is uniformly distributed. When a user has set the instant to sense the spectrum within the sensing process, it is repeated every $T_{P}$ sec

In the example, channels to be sensed are randomly selected with equal probability. Consequently, $W$ becomes a binomial random variable with

$$
P(W=w)=P_{\text {select }} \cdot\left(1-P_{\text {select }}\right)^{w-1}
$$

where $P_{\text {select }}$ is the probability that a particular channel is selected in a sensing process. If all the channels are selected with the same probability, $P_{\text {select }}=\frac{\phi_{T}}{|M|}$.

The number of primary channels is $|M|=20$ and the probability that a secondary node senses an available channel, $P_{\text {idle }}$, has been obtained from cellular systems $[10,11]$. Based on these studies the spectrum bands present an occupancy around $25 \%$, therefore $P_{\text {idle }}=0.75$

With regard to the duration of the reliability of the collected spectrum information, $T_{U}$, its value has been obtained from the traffic model of UMTS [12]. The most stringent constraint is put forward by traffic with bursty nature, since the more variable traffic is, the less accurate to assume the reliability of the information is. According to [12], the time between packets in non-real time services is $0.5 \mathrm{sec}$., therefore $T_{U}=0.5 \mathrm{sec}$. This value agrees with the values used, for instance, in [13].
The time devoted to sense a channel, $T_{C h \text { Sensed }}$, has been determined with the false alarm-sensing time curve in [14]. In order to have an approximate false alarm of $10 \%, T_{C h \text { Sensed }}=4.5$ msec. Furthermore, the time to switch from one channel to another is $T_{\text {switch }}=200 \mu$ sec [15] and the time for PHY and MAC header transmission is $T_{\text {header }}=368 \mu$ sec [16]. It is also assumed that the information on one channel is transmitted in 2 bytes, i.e. $T_{\text {channel }}=0.148 \mu \mathrm{sec}$, and that no additional information is piggybacked in the packet, $T_{\text {other }}=0 \mathrm{sec}$.

Figure 2 shows, for a given $\left|V_{i}\right|$ and $\phi_{T}$, the minimum required sensing period $T_{P}$ to have $\bar{I}_{i}=2$ and $\bar{I}_{i}=3$. As expected, $T_{P}$ grows as $\bar{I}_{i}$ is decreased. It is worth noting that the relationship between $T_{P}$ and $\bar{I}_{i}$ has a huge impact on the design of any cooperative MAC protocol for cognitive networks, since it determines the amount of available resources, in terms of frequency channels, and the cost to obtain them. On the other hand, the model allows quantifying the tradeoff between the cost of sensing a certain number of channels and the benefit obtained.

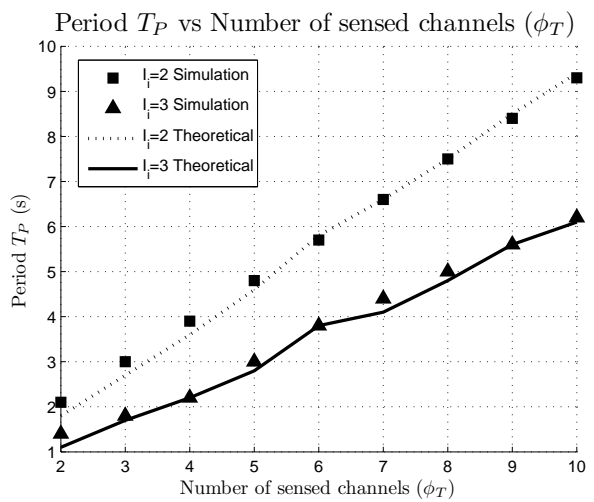

Figure 2: Minimum $T_{P}$ required for $\bar{I}_{i}=2$ and $\bar{I}_{i}=3$ with $\left|V_{i}\right|=4$.

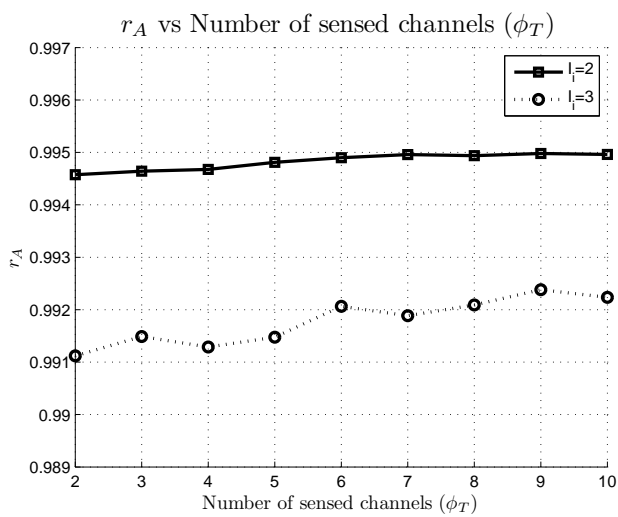

Figure 3: Normalized available time for data.

In Figure 3, the $r_{A}$ associated to the $T_{P}$ values obtained in Figure 2 are shown. It is worth noting that $r_{A}$ is approximately 1 , the necessary condition for the model to be valid. Figure 2 and Figure 3 imply that, for a given design $\bar{I}_{i}$, there are several $\left(\phi_{T}, T_{P}\right)$ possible pairs (one for each $\phi_{T}$ while $\left.T_{\text {Sensing }}<T_{P}\right)$. Although $r_{A}$ is very stable for any 
$\phi_{T}$, Figure 3 suggests that high $\phi_{T}$ values offer better performance. Notice, however, that despite the fact that the average number of available channels, $\bar{I}_{i}$, is kept in both figures, the variance of $I_{i}$ rises as $T_{P}$ grows. Therefore, excessively high $\phi_{T}$ values should also be avoided.

\section{CONCLUSIONS}

The MAC layer cooperative sensing in cognitive networks has been modeled and evaluated in this paper. When nodes are equipped with a single transciever, the available time must be shared between the sensing process and the data transmission. Furthermore, it is important to reduce the time devoted to sensing while guaranteeing a minimum number of monitored available channels. The obtained results show that the cooperation among SUs leads to less frequent sensing processes. Likewise, the number of sensed channels during each sensing process also impacts on the sensing period.

\section{REFERENCES}

[1] ITU-R, Radio Regulations. 2004.

[2] Federal Communications Commission, Spectrum Policy Task Force, Rep. ET Docket No. 02-135. 2002.

[3] T. Y. et al. A survey of spectrum sensing algorithms for cognitive radio applications. IEEE Communications Surveys $\&$ Tutorials, 11(1):116-130, 2009.

[4] A. H. et al. Adaptive scheduling of spectrum sensing periods in cognitive radio networks. IEEE GLOBECOM, pages 3128-3132, 2007.

[5] J. J. et al. HC-MAC: A hardware-constrained cognitive mac for efficient spectrum management. IEEE GLOBECOM, 6:106-117, 2007.

[6] Z. J. et al. Distributed coordination in dynamic spectrum allocation networks. IEEE DySPAN, pages 259-268, 2005.

[7] T. C. et al. Cogmesh: A cluster-based cognitive radio network. IEEE DySPAN, pages 168-178, 2007.

[8] L. L. et al. OSA-mac: A mac protocol for opportunistic spectrum access in cognitive radio networks. IEEE $W C N C$, pages 1426-1430, 2008.

[9] L. Z. et al. A slot-based mac protocol in cognitive wireless radio networks. WiCOM, pages 1-4, 2008.

[10] A. G. et al. Spectrum sensing in cognitive radio networks: Requirement, challenges and design trade-off. IEEE Communications Magazine, 46(4):32-39

[11] M. H. I. et al. Spectrum survey in singapore: Occupancy measurements and analyses. CrownCom, pages $1-7,2008$.

[12] 3GPP TR 30.03U. Selection procedures for the choice of radio transmission technologies of the umts. IEEE Communications Magazine, March 1998.

[13] H. K. et al. Efficient discovery of spectrum opportunities with mac-layer sensing in cognitive radio networks. IEEE Transaction on Mobile Computing, $7(5): 533-545,2008$.

[14] Y. C. L. et al. Sensing throughput tradeoff for cognitive radio networks. IEEE Transactions on Wireless Communications, 7(4), 2008.

[15] K. R. C. et al. Cognitive wireless mesh networks with dynamic spectrum access. IEEE Journal on Selected Areas in Communications, 26(1):168-181, 2008.

[16] Ieee Std. 802.11g. Supplement to part 11: Wireless lan medium access control (mac) and physical layer (phy) specifications; further high-speed physical layer extension in the $2.4 \mathrm{Ghz}$ band. 